\title{
Determination of atom-field observables via resonant interaction
}

\author{
A. Luis and L. L. Sánchez-Soto \\ Departamento de Óptica, Facultad de Ciencias Físicas, Universidad Complutense, 28040 Madrid, Spain
}

(Received 22 October 1997)

\begin{abstract}
We find the observables that can be determined by two practical schemes based on atom-field resonant interaction. In the first example, the interaction is followed by photon-number and atomic-population measurements. In the second scheme the directly measured quantity is the atomic deflection. In particular, we show that they provide the measurement of the atom-field relative phase. When the initial field state is known, the atomic density matrix can be reconstructed. [S1050-2947(98)10704-7]
\end{abstract}

PACS number(s): 42.50.Dv, 03.65.Bz, 42.50.Vk

\section{INTRODUCTION}

Quantum mechanics postulates that every self-adjoint operator can be measured (leaving aside superselection rules). This also applies to positive-operator measures through their Naimark extension [1]. Although the practical implementation of such measurements is known in very few cases, there are methods extending the number of observables whose statistics can be determined in practice. For instance, controllable couplings can relate directly measurable quantities with more involved observables through a suitable data analysis. The dependence of the statistics on controllable parameters is the basis of generalized measurements described by positive-operator measures. This also underlies well-known schemes determining the quantum state, like tomography, for instance [2].

In this work we reexamine this issue for the measurement of observables of a two-level atom and a one-mode electromagnetic field. Two simple arrangements based on resonant interaction are considered. In the first example, resonant interaction is followed by the measurement of photon number and atomic population. In the second arrangement, the measured observable is the atomic deflection $[3,4]$. Both schemes provide information about the atom-field state prior to the coupling.

The plan of this work is as follows: In Secs. II and III, we analyze the joint atom-field observables that can be determined from the statistics of the corresponding measurement. In Sec. IV, we consider the possibility of measuring the atom-field relative phase $[5,6]$, including a concrete example to illustrate the experimental feasibility of the method. Resonant interaction is highly sensitive to phase relations between the field and the atomic dipole. The output measurable quantities depend on the initial relative phase, so the arrangements studied here should allow us to determine the quantum statistics of this observable. Given the relevance of phase variables and the difficulties that their quantum description encounter, we think it is worth examining the possibility of their practical measurement [7].

If the initial field state is known, these arrangements can be regarded as generalized measurements of atomic observables. In Sec. V we study the atomic variables that can be determined in this way. The possibility of reconstructing the density matrix of the atom is examined as well.

\section{DETERMINATION OF ATOM-FIELD OBSERVABLES BY PHOTON-NUMBER AND ATOMIC-POPULATION MEASUREMENTS}

In this section we examine the observables that can be determined when atom-field resonant interaction is followed by the simultaneous measurement of field-photon number and atomic-level population. The interaction is conveniently described by the Jaynes-Cummings Hamiltonian in the rotating-wave approximation (in units $\hbar=1$ ) [8]

$$
H=\omega\left(S_{z}+a^{\dagger} a\right)+\lambda\left(a^{\dagger} S_{-}+a S_{+}\right),
$$

where $\quad S_{z}=(|e\rangle\langle e|-| g\rangle\langle g|) / 2, \quad S_{+}=|e\rangle\langle g|, \quad$ and $S_{-}=|g\rangle\langle e|$. The vectors $|e\rangle$ and $|g\rangle$ represent the excited and ground energy levels of the isolated atom, $a$ and $a^{\dagger}$ are the annihilation and creation operators for the field mode, and $\lambda$ is the coupling constant. For simplicity, exact resonance has been assumed.

We shall consider that the field experiences a controllable phase shift $\delta$ before its interaction with the atom, which is equivalent to shift by $-\delta$ the atomic phase. If $\rho_{\text {in }}$ is the initial density matrix of the atom-field system, the final output density matrix $\rho_{\text {out }}$ after these two steps can be written as

$$
\rho_{\text {out }}=U \rho_{\text {in }} U^{\dagger},
$$

where the unitary operator $U$ is

$$
U=e^{-i \tau H} e^{i \delta a^{\dagger} a}
$$

and $\tau$ is the interaction time.

The joint probability for the simultaneous measurement of photon number and atomic population is

$$
P_{\text {out }}(j, n, \delta)=\operatorname{tr}\left(\rho_{\text {out }}|j, n\rangle\langle j, n|\right),
$$

where $j=e, g$. Since only one fixed interaction time $\tau$ will be needed, $\tau$ is not included in the parametrization of these probabilities.

Our purpose is to extract from this measurement the statistics of atom-field variables in the initial state. To this end, we express the probabilities (2.4) in terms of $\rho_{\text {in }}$,

$$
P_{\text {out }}(j, n, \delta)=\operatorname{tr}\left(\rho_{\text {in }} U^{\dagger}|j, n\rangle\langle j, n| U\right)=\operatorname{tr}\left[\rho_{\text {in }} \Delta(j, n, \delta)\right],
$$


where

$$
\begin{aligned}
\Delta(g, n, \delta)= & c_{n}^{2}|g, n\rangle\left\langle g, n\left|+s_{n}^{2}\right| e, n-1\right\rangle\langle e, n-1| \\
& +i s_{n} c_{n}\left(e^{i \delta}|e, n-1\rangle\langle g, n|\right. \\
& \left.-e^{-i \delta}|g, n\rangle\langle e, n-1|\right), \\
\Delta(e, n, \delta)= & s_{n+1}^{2}|g, n+1\rangle\left\langle g, n+1\left|+c_{n+1}^{2}\right| e, n\right\rangle\langle e, n| \\
& -i s_{n+1} c_{n+1}\left(e^{i \delta}|e, n\rangle\langle g, n+1|\right. \\
& \left.-e^{-i \delta}|g, n+1\rangle\langle e, n|\right),
\end{aligned}
$$

and

$$
\begin{aligned}
& s_{n}=\sin (\lambda \tau \sqrt{n}), \\
& c_{n}=\cos (\lambda \tau \sqrt{n})
\end{aligned}
$$

are known parameters.

The determination of atom-field observables requires the inversion of the previous equations, expressing $|e, n\rangle\langle e, n|$, $|g, n\rangle\langle g, n|| e,, n-1\rangle\langle g, n|$, and $|g, n\rangle\langle e, n-1|$ as functions of $\Delta(e, n, \delta)$ and $\Delta(g, n, \delta)$. This inversion can be carried out by means of a discrete Fourier analysis in $\delta$. In this case three values for $\delta$ are enough, for instance $\delta_{r}=2 \pi r / 3$ with $r=0, \pm 1$. This gives the following equations:

$$
\begin{aligned}
& |e, n\rangle\langle e, n|=\frac{1}{2}[\Delta(e, n, \delta)+\Delta(g, n+1, \delta)] \\
& +\frac{1}{6 c_{4 n+4}} \sum_{r}\left[\Delta\left(e, n, \delta_{r}\right)-\Delta\left(g, n+1, \delta_{r}\right)\right], \\
& |g, n\rangle\langle g, n|=\frac{1}{2}[\Delta(e, n-1, \delta)+\Delta(g, n, \delta)] \\
& -\frac{1}{6 c_{4 n}} \sum_{r}\left[\Delta\left(e, n-1, \delta_{r}\right)-\Delta\left(g, n, \delta_{r}\right)\right], \\
& |g, 0\rangle\langle g, 0|=\Delta(g, 0),
\end{aligned}
$$

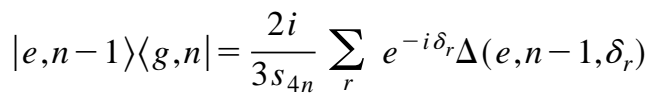

$$
\begin{aligned}
& =\frac{-2 i}{3 s_{4 n}} \sum_{r} e^{-i \delta_{r} \Delta}\left(g, n, \delta_{r}\right),
\end{aligned}
$$

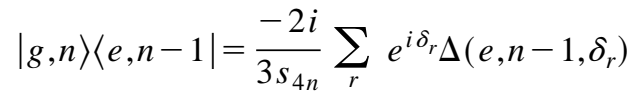

$$
\begin{aligned}
& =\frac{2 i}{3 s_{4 n}} \sum_{r} e^{i \delta_{r} \Delta}\left(g, n, \delta_{r}\right) \text {. }
\end{aligned}
$$

It should be noted that $[\Delta(e, n-1, \delta)+\Delta(g, n, \delta)]$ and $\Delta(g, 0)$ are independent of $\delta$, so any $\delta_{r}$ can be used whenever they appear. We have assumed that the interaction time $\tau$ is chosen such that $s_{4 n}=\sin (2 \lambda \tau \sqrt{n}) \neq 0$ and $c_{4 n}=\cos (2 \lambda \tau \sqrt{n}) \neq 0$.
Thus Eqs. (2.8) provide the statistics of any observable expressible as an arbitrary linear combination of the operators

$$
\begin{aligned}
|e, n\rangle\langle e, n|, \quad & |g, n\rangle\langle g, n|, \quad| e, n-1\rangle\langle g, n|, \\
& |g, n\rangle\langle e, n-1|,
\end{aligned}
$$

i.e., of every observable commuting with $S_{z}+a^{\dagger} a$. To this end, probabilities (2.5) should be known for the three values $\delta_{r}$ of the phase shift. This can be accomplished by repeating the measurement after each phase shift $\delta_{r}$. In addition, this can be achieved in the form of a single generalized measurement. The three phase shifts can be embodied in a single realization by using a nonresonant interaction of the field with an auxiliary three-level atom, for example [9]. One of the levels can be too strongly detuned to affect or be affected by the field, while the other two can be sufficiently detuned so that the transition probability is negligible. This nonresonant interaction produces a phase shift in the field that depends on the atomic level. If the auxiliary atom is prepared in a superposition of its three states, the detection of the energy level after the interaction implies the corresponding phase shift in the field, which can be adjusted to be $\delta_{r}, r=0 \pm 1$, by properly selecting the detuning, coupling constant, and interaction time.

Before considering particular examples, we can express the result obtained in a slightly different form. The measurement provides information about the atom-field state $\rho_{\text {in }}$. Although this information is not complete, we can nevertheless express this partial knowledge in terms of a quasiprobability distribution in phase space, for instance the $Q$ function. The atom-field $Q$ function is defined as

$$
Q(\alpha, \vartheta, \varphi)=\frac{1}{2 \pi^{2}} \operatorname{tr}\left(\rho_{\text {in }}|\vartheta, \varphi\rangle|\alpha\rangle\langle\alpha|\langle\vartheta, \varphi|\right)
$$

where $|\alpha\rangle$ are field coherent states, and

$$
|\vartheta, \varphi\rangle=\sin (\vartheta / 2)|g\rangle+e^{i \varphi} \cos (\vartheta / 2)|e\rangle,
$$

are SU(2) coherent states for the atom [10]. Instead of the complete $Q(\alpha, \vartheta, \varphi)$ function, the measurement provides a reduced $Q$ function depending just on $|\alpha|, \vartheta$, and the phase difference $\phi=\varphi-\theta$ between the atomic phase $\varphi$ and the field phase $\theta$ :

$$
q(|\alpha|, \vartheta, \phi)=\int d \theta Q\left(\alpha=|\alpha| e^{i \theta}, \vartheta, \varphi=\phi+\theta\right)
$$

This is because

$$
\int d \theta|\vartheta, \varphi=\phi+\theta\rangle|\alpha=| \alpha\left|e^{i \theta}\right\rangle\left\langle\alpha=|\alpha| e^{i \theta}\right|\langle\vartheta, \varphi=\phi+\theta|
$$

commutes with $S_{z}+a^{\dagger} a$, so Eqs. (2.8) can be used to express $q(|\alpha|, \vartheta, \phi)$ in terms of the statistics of the measurement. Similar results would be obtained by using other quasiprobability distributions.

This phase-space picture expresses the main result of this section in a more classical way. The atom-field resonant cou- 
pling backtransforms $\vartheta$ and $|\alpha|$ (which are phase-space counterparts of the measured operators $S_{z}$ and $a^{\dagger} a$ ) into functions of $\vartheta,|\alpha|$, and $\phi$. This, and the determination of $q(|\alpha|, \vartheta, \phi)$ just shown, suggest that the quantum translation of the relative phase should be included among the observables whose statistics can be derived from this measuring scheme. Section IV is devoted to an examination of this possibility in purely quantum terms.

\section{DETERMINATION OF ATOM-FIELD OBSERVABLES VIA ATOMIC DEFLECTION}

Here we analyze a different measurement scheme based also on the resonant coupling of the atom with a single-mode field. In this case, we shall consider the atomic deflection produced when the atom passes near the node of a standing electromagnetic wave [4]. The information about atom-field variables will be contained in the change of transverse momentum $\wp$ experienced by the atom.

As in Sec. II, we assume that, before the atom-field interaction, a controllable phase shift $\delta$ can be produced on the field. Afterwards, the atom crosses a standing light field aligned along the $X$ direction, passing through a small transverse region centered around a node at $x=0$. If this region is small enough, the interaction can be described by the Hamiltonian

$$
H_{\mathrm{int}}=-\kappa x\left(a^{\dagger} S_{-}+a S_{+}\right)
$$

where $\kappa$ is a coupling constant and $x$ denotes the corresponding position for the center of mass of the atom. We assume the Raman-Nath regime [11], where the motion of the atom along $X$ during its passage through the standing wave may be ignored, and the kinetic energy in the Hamiltonian can be neglected. In this limit there is a transverse momentum shift associated with each eigenvalue of $\kappa\left(a^{\dagger} S_{-}+a S_{+}\right)$. The output transverse-momentum distribution $\mathcal{P}_{\text {out }}(\wp, \delta)$ for a given field-phase shift $\delta$ is

$\mathcal{P}_{\text {out }}(\wp, \delta)=P_{\text {in }}(0) \mathcal{P}_{\text {in }}(\wp)+\sum_{n=1, \pm}^{\infty} P_{\text {in }}^{( \pm)}(n, \delta) \mathcal{P}_{\text {in }}(\wp \mp \kappa \tau \sqrt{n})$,

where $\mathcal{P}_{\text {in }}(\wp)$ is the initial distribution, $\tau$ is the interaction time,

$$
\begin{gathered}
P_{\text {in }}(0)=\operatorname{tr}\left[\rho_{\text {in }} \Delta(0)\right], \\
P_{\text {in }}^{( \pm)}(n, \delta)=\operatorname{tr}\left[\rho_{\text {in }} \Delta^{( \pm)}(n, \delta)\right],
\end{gathered}
$$

with

$$
\begin{gathered}
\Delta(0)=|g, 0\rangle\langle g, 0|, \\
\Delta^{( \pm)}(n, \delta)=\frac{1}{2}(|g, n\rangle\langle g, n|+| e, n-1\rangle\langle e, n-1| \\
\left. \pm e^{i \delta}|e, n-1\rangle\left\langle g, n\left| \pm e^{-i \delta}\right| g, n\right\rangle\langle e, n-1|\right),
\end{gathered}
$$

and $\rho_{\text {in }}$ is the initial density matrix representing the field and the internal state of the atom. We have assumed that initially $\wp$ is uncorrelated with the rest of variables. The output probabilities $\mathcal{P}_{\text {out }}(\wp, \delta)$ can be measured by observing the spatial distribution of the atom far away from the standing field. For simplicity, we consider a Gaussian for $\mathcal{P}_{\text {in }}(\wp)$,

$$
\mathcal{P}_{\text {in }}(\wp)=\frac{1}{\sqrt{2 \pi} \sigma_{\wp}} \exp -\left(\frac{\wp^{2}}{2 \sigma_{\wp}^{2}}\right) .
$$

If $\kappa \tau / \sigma_{\wp}$ is large enough, the output distribution consists of separate peaks centered at the values

$$
\wp= \pm \kappa \tau \sqrt{n} .
$$

According to Eq. (3.2), we have $\mathcal{P}_{\text {out }}(\wp= \pm \kappa \tau \sqrt{n}, \delta)$ $\propto P_{\text {in }}^{( \pm)}(n, \delta)$, so the peak heights at these points give directly the probabilities (3.3).

As in Sec. II the knowledge of these probabilities for three phase shifts, $\delta_{r}=2 \pi r / 3, r=0, \pm 1$, allows us to infer the statistics of any linear combination of

$$
\begin{gathered}
|g, n\rangle\langle g, n|+| e, n-1\rangle\langle e, n-1|, \quad| g, 0\rangle\langle g, 0|, \\
|e, n-1\rangle\langle g, n|, \quad| g, n\rangle\langle e, n-1|
\end{gathered}
$$

by inverting Eq. (3.4) with the help of a discrete Fourier analysis.

The set of observables that can be determined now is smaller than before, since the separate contribution of the projectors $|g, n\rangle\langle g, n|$ and $|e, n-1\rangle\langle e, n-1|$ is not available without further measurements. Nevertheless, we shall show in Sec. IV that this scheme also provides the measurement of the phase difference between the atomic dipole and the field.

\section{MEASUREMENT OF THE ATOM-FIELD RELATIVE PHASE}

We have shown that the statistics of observables commuting with $S_{z}+a^{\dagger} a$ can be derived from the probabilities of these measurements. This must include the atom-field phase difference. The operator $S_{z}+a^{\dagger} a$ generates identical shifts in the field and atomic-dipole phases, leaving the phase difference unchanged. Then the quantum description of the relative phase must commute with $S_{z}+a^{\dagger} a$.

Besides this general remark, it is still necessary to specify what may be understood as the quantum translation of this variable. Because of the problematic description of phase, several different approaches are available. Two main possibilities have been examined recently $[5,6]$. It has been shown that a unitary operator $E_{\phi}$ representing the exponential of the phase difference and commuting with $S_{z}+a^{\dagger} a$ is defined by the polar decomposition [5]

$$
S_{-} a^{\dagger}=\sqrt{S_{-} S_{+} a^{\dagger} a} E_{\phi}=E_{\phi} \sqrt{S_{+} S_{-} a a^{\dagger}} .
$$

The simultaneous eigenvectors of $E_{\phi}$ and $S_{z}+a^{\dagger} a$ are

$$
\begin{gathered}
\left|\phi_{0}^{(0)}\right\rangle=|g, 0\rangle, \\
\left|\phi_{ \pm}^{(n)}\right\rangle=\frac{1}{\sqrt{2}}(|g, n\rangle \pm i|e, n-1\rangle),
\end{gathered}
$$

with eigenvalues 


$$
\begin{gathered}
E_{\phi}\left|\phi_{0}^{(0)}\right\rangle=\left|\phi_{0}^{(0)}\right\rangle \\
E_{\phi}\left|\phi_{ \pm}^{(n)}\right\rangle= \pm i\left|\phi_{ \pm}^{(n)}\right\rangle \\
\left(S_{z}+a^{\dagger} a\right)\left|\phi^{(n)}\right\rangle=\left(n-\frac{1}{2}\right)\left|\phi^{(n)}\right\rangle .
\end{gathered}
$$

These eigenvectors define a joint probability distribution for the phase difference and $S_{z}+a^{\dagger} a$ as

$$
\begin{aligned}
& P_{\text {in }}\left(0, \phi_{0}^{(0)}\right)=\operatorname{tr}\left(\rho_{\text {in }}\left|\phi_{0}^{(0)}\right\rangle\left\langle\phi_{0}^{(0)}\right|\right), \\
& P_{\text {in }}\left(n, \phi_{ \pm}^{(n)}\right)=\operatorname{tr}\left(\rho_{\text {in }}\left|\phi_{ \pm}^{(n)}\right\rangle\left\langle\phi_{ \pm}^{(n)}\right|\right) .
\end{aligned}
$$

Focusing on the scheme analyzed in Sec. II, we have that the orthogonal projectors (4.4) are linear combinations of the operators (2.9). Equations (2.8) give

$$
\begin{aligned}
& \left|\phi_{0}^{(0)}\right\rangle\left\langle\phi_{0}^{(0)}\right|=\Delta(g, 0), \\
& \left|\phi_{ \pm}^{(n)}\right\rangle\left\langle\phi_{ \pm}^{(n)}\right|=\frac{1}{2}[\Delta(e, n-1, \delta)+\Delta(g, n, \delta)] \\
& \mp \frac{1}{3 s_{4 n}} \sum_{r}\left[e^{-i \delta_{r} \Delta}\left(e, n-1, \delta_{r}\right)\right.
\end{aligned}
$$

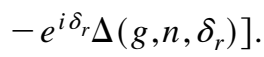

Then, the statistics of the phase-difference operator can be extracted from the measured probabilities $P_{\text {out }}(j, n, \delta)$.

Similarly, the scheme examined in Sec. III can be used for this purpose, since the projection measure in Eq. (4.4) is a linear combination of the operators (3.7). This shows that this operator is indirectly measured in both schemes.

Nevertheless, the arrangement based on atomic deflection also allows the direct measurement of this phase-difference operator. To this end, the parameter $\delta$ in Sec. III can be chosen to be $\delta=\pi / 2$. In such a case, the transverse momentum distribution at the peaks is proportional to the phasedifference probabilities (4.4) since, from Eq. (3.4), we have

$$
\Delta^{( \pm)}(n, \delta=\pi / 2)=\left|\phi_{ \pm}^{(n)}\right\rangle\left\langle\phi_{ \pm}^{(n)}\right|
$$

and then

$$
\mathcal{P}_{\text {out }}(\wp= \pm \kappa \tau \sqrt{n}, \delta=\pi / 2) \propto P_{\text {in }}\left(n, \phi_{ \pm}^{(n)}\right) .
$$

Besides the operator description, there is also the possibility of describing the phase difference by taking the marginal distribution for this variable from a joint positiveoperator measure for the dipole and field absolute phases $[6,7,12]$. The result is a positive-operator measure $\Lambda(\phi)$ commuting with $S_{z}+a^{\dagger} a$,

$$
\Lambda(\phi)=\sum_{n=0}^{\infty} \Lambda(n, \phi)
$$

where $\Lambda(n, \phi)$ has the general form

$$
\begin{gathered}
\Lambda(0, \phi)=\frac{1}{2 \pi}|g, 0\rangle\langle g, 0|, \\
\Lambda(n, \phi)=\frac{1}{2 \pi}(|e, n-1\rangle\langle e, n-1|+| g, n\rangle\langle g, n| \\
\left.+\mu_{n} e^{i \phi}|e, n-1\rangle\left\langle g, n\left|+\mu_{n}^{*} e^{-i \phi}\right| g, n\right\rangle\langle e, n-1|\right),
\end{gathered}
$$

and $\mu_{n}$ are parameters depending on the particular approach used for the absolute phases. This positive-operator measure is again a linear combination of the operators (2.9) or (3.7), so the corresponding probability distribution can be deduced from the statistics of the measurements in Secs. II and III.

The preceding calculations show how the atom-field relative phase can be theoretically inferred from measurement. However, this inference will be affected by the limited accuracy attainable in real measurements. In the case of a practical realization of the arrangement examined is Sec. II, there are several sources of uncertainty such as detection inefficiencies or the spread of the interaction time, for instance. Therefore, it would be desirable to examine in which way the reconstructed values would deviate from the true input values under realistic practical conditions.

The consequences of nonunit detection efficiencies and the way they can be dealt with have been well studied [13], so we will consider in some detail the effect of an imprecise determination of the interaction time. We can see in Eqs. (4.5) or (4.9) that the interaction time appears only when obtaining the nondiagonal matrix elements $\left\langle e, n-1\left|\rho_{\text {in }}\right| g, n\right\rangle$ from Eqs. (2.8). For instance, we have

$$
\frac{2 i}{3} \sum_{r} e^{i \delta_{r}} P_{\text {out }}\left(g, n, \delta_{r}\right)=s_{4 n}\left\langle e, n-1\left|\rho_{\text {in }}\right| g, n\right\rangle .
$$

To calculate the deviation of the inferred values from the true ones, we will assume a Gaussian distribution of possible interaction times,

$$
W\left(\tau^{\prime}\right)=\frac{1}{\sqrt{2 \pi} \sigma_{\tau}} \exp -\frac{\left(\tau^{\prime}-\tau\right)^{2}}{2 \sigma_{\tau}^{2}}
$$

with average interaction time $\tau$ and width $\sigma_{\tau}$. The measured values $\left\langle e, n-1\left|\rho_{\text {in }}\right| g, n\right\rangle_{\text {meas }}$ are obtained after averaging $P_{\text {out }}\left(g, n, \delta_{r}\right)$ over the distribution $W\left(\tau^{\prime}\right)$, leading to

$$
\begin{aligned}
& \left\langle e, n-1\left|\rho_{\text {in }}\right| g, n\right\rangle_{\text {meas }}=\frac{1}{s_{4 n}} \frac{2 i}{3} \sum_{r} e^{i \delta_{r}} \bar{P}_{\text {out }}\left(g, n, \delta_{r}\right) \\
& =\frac{\bar{s}_{4 n}}{s_{4 n}}\left\langle e, n-1\left|\rho_{\text {in }}\right| g, n\right\rangle,
\end{aligned}
$$

where $\bar{P}_{\text {out }}\left(g, n, \delta_{r}\right)$ and $\bar{s}_{4 n}$ denote the corresponding time averages. Since

$$
\bar{s}_{4 n}=s_{4 n} e^{-2 \lambda^{2} \sigma_{\tau}^{2} n}
$$

we have the following relation between inferred and true values 


$$
\left\langle e, n-1\left|\rho_{\text {in }}\right| g, n\right\rangle_{\text {meas }}=e^{-2 \lambda^{2} \sigma_{\tau}^{2} n}\left\langle e, n-1\left|\rho_{\text {in }}\right| g, n\right\rangle .
$$

This relation leads to a measured probability distribution for the relative phase which is broader and smoother than the true one. This is the deteriorating effect of the spread of interaction times.

In principle, this effect can be numerically compensated for, provided that the distribution $W\left(\tau^{\prime}\right)$ were known. However, such compensation will increase the effect of an unavoidably imprecise experimental determination of the probabilities $P_{\text {out }}\left(g, n, \delta_{r}\right)$.

Next we evaluate relation (4.14) under realistic practical values for the parameters involved. We will see that, in fact, this can be a rather small effect. In principle, any average interaction time $\tau$ is valid provided that $s_{4 n} \neq 0$, so that Eq. (4.10) can be inverted. However, from a more practical perspective, the optimum value will be the one leading to maximum $s_{4 n}$, in order to minimize the effect of experimental noise in $P_{\text {out }}\left(g, n, \delta_{r}\right)$ and the lack of a precise knowledge of $\tau$. If the field state has a mean photon number $\bar{n}$, we can impose $s_{4 \bar{n}} \sim 1$, which means $\lambda \tau \sqrt{\bar{n}} \sim 1$. Then Eq. (4.14) can be written as

$$
\left\langle e, n-1\left|\rho_{\text {in }}\right| g, n\right\rangle_{\text {meas }}=e^{-2(n / \bar{n})\left(\sigma_{\tau} / \tau\right)^{2}}\left\langle e, n-1\left|\rho_{\text {in }}\right| g, n\right\rangle .
$$

Accuracies in the interaction time of the order of $1 \%$ are within the current experimental values, so that $\left(\sigma_{\tau} / \tau\right)^{2} \sim 10^{-4}$, and the exponential in Eq. (4.15) is of the order of unity. To be more specific, we can consider a micromaser configuration: for Rydberg atoms the coupling constant $\lambda$ can be of the order of $10^{4}-10^{6} \mathrm{~s}^{-1}$, and mean atomic velocities are in the range $10^{1}-10^{3} \mathrm{~m} / \mathrm{s}$, with a velocity spread of $1 \%$. This leads to average interaction times ranging from $10^{-5}$ to $10^{-3} \mathrm{~s}$ with a time spread $\sigma_{\tau} / \tau \sim 0.01[8,9,14]$. With this typical values, the optimum $\lambda \tau \sqrt{\bar{n}} \sim 1$ can be satisfied even in the case of very small photon numbers. Therefore, we conclude that under actual experimental conditions the deviation of the inferred values from the true ones is not noticeable.

This discussion could be extended also to the arrangement analyzed in Sec. III. In such a case the interaction time and its spread will affect the position and width, respectively, of the peaks of the spatial distribution of atoms far away from the standing field. Each peak at $\wp= \pm \kappa \tau \sqrt{n}$ will have a width $\sigma_{\wp}^{2}+\left(\kappa \sigma_{\tau}\right)^{2} n$. A numerical evaluation using parameters within the preceding ranges and previously considered values for $\sigma_{\wp}$ [4] shows that for small photon numbers the corresponding peaks are clearly distinguishable, even after including the interaction-time spread.

\section{QUANTUM ATOMIC-STATE RECONSTRUCTION}

So far we have been concerned with a determination of atom-field variables assuming that before the measurement the density matrix is completely unknown. It can be interesting to examine what happens when the initial system state factorizes $\rho_{\text {in }}=\rho_{\text {in }}^{a} \otimes \rho_{\text {in }}^{f}$, and the initial field state $\rho_{\text {in }}^{f}$ is known. In such a case, the information supplied by the mea- surement can be regarded as information about the atomic state. In other words, this leads to a generalized measurement of the atomic system. We shall study the atomic observables that can be determined by using the arrangement of Sec. II, for instance.

The knowledge of the initial field state can be taken into account by performing the trace over the field variables in Eq. (2.5). This leads to a positive-operator measure in the Hilbert space of the atom

$$
\Delta^{a}(j, n, \delta)=\operatorname{tr}_{f}\left[\rho_{\text {in }}^{f} \Delta(j, n, \delta)\right],
$$

and the statistics of the measurement can be regarded as depending only on the atomic state

$$
P_{\text {out }}(j, n, \delta)=\operatorname{tr}_{a}\left[\rho_{\text {in }}^{a} \Delta^{a}(j, n, \delta)\right] .
$$

This atomic positive-operator measure is given by

$$
\begin{gathered}
\Delta^{a}(g, n, \delta)=c_{n}^{2} \rho_{n, n}|g\rangle\left\langle g\left|+s_{n}^{2} \rho_{n-1, n-1}\right| e\right\rangle\langle e| \\
+i s_{n} c_{n}\left(e^{i \delta} \rho_{n, n-1}|e\rangle\left\langle g\left|-e^{-i \delta} \rho_{n-1, n}\right| g\right\rangle\langle e|\right) \\
\Delta^{a}(e, n, \delta)= \\
s_{n+1}^{2} \rho_{n+1, n+1}|g\rangle\left\langle g\left|+c_{n+1}^{2} \rho_{n, n}\right| e\right\rangle\langle e| \\
-i s_{n+1} c_{n+1}\left(e^{i \delta} \rho_{n+1, n}|e\rangle\langle g|\right. \\
\left.-e^{-i \delta} \rho_{n, n+1}|g\rangle\langle e|\right),
\end{gathered}
$$

where the field matrix elements $\rho_{n, n^{\prime}}=\left\langle n\left|\rho_{\text {in }}^{f}\right| n^{\prime}\right\rangle$ are known quantities.

As a matter of fact, the photon-number variable carries no relevant information, so we can remove it by summing over $n$,

$$
\begin{aligned}
\Delta^{a}(g, \delta)= & \sum_{n=0}^{\infty} \Delta^{a}(g, n, \delta)=c|g\rangle\langle g|+s| e\rangle\langle e| \\
& +\frac{i}{2}\left(e^{i \delta} d|e\rangle\left\langle g\left|-e^{-i \delta} d^{*}\right| g\right\rangle\langle e|\right), \\
\Delta^{a}(e, \delta) & =\sum_{n=0}^{\infty} \Delta^{a}(e, n, \delta)=I-\Delta^{a}(g, \delta),
\end{aligned}
$$

where $I$ is the identity in the atomic Hilbert space, and the parameters $c, s$, and $d$ are

$$
\begin{gathered}
c=\sum_{n=0}^{\infty} \cos ^{2}(\lambda \tau \sqrt{n})\left\langle n\left|\rho_{\mathrm{in}}^{f}\right| n\right\rangle, \\
s=\sum_{n=0}^{\infty} \sin ^{2}(\lambda \tau \sqrt{n+1})\left\langle n\left|\rho_{\mathrm{in}}^{f}\right| n\right\rangle, \\
d=\sum_{n=0}^{\infty} \sin (2 \lambda \tau \sqrt{n+1})\left\langle n+1\left|\rho_{\mathrm{in}}^{f}\right| n\right\rangle .
\end{gathered}
$$

This corresponds to perform no measurement on the field. After the interaction only the atomic level is detected.

Equations (5.4) can be inverted following the same procedure of preceding sections and, provided that $d \neq 0$ and $c$ $\neq s$, we have 


$$
\begin{gathered}
|e\rangle\langle e|=\frac{1}{3(s-c)}\left[\sum_{r} \Delta^{a}\left(g, \delta_{r}\right)-3 c I\right], \\
|g\rangle\langle g|=\frac{1}{3(c-s)}\left[\sum_{r} \Delta^{a}\left(g, \delta_{r}\right)-3 s I\right], \\
|e\rangle\langle g|=\frac{-2 i}{3 d} \sum_{r} e^{-i \delta_{r} \Delta^{a}\left(g, \delta_{r}\right),} \\
|g\rangle\langle e|=\frac{2 i}{3 d^{*}} \sum_{r} e^{i \delta_{r} \Delta^{a}\left(g, \delta_{r}\right) .}
\end{gathered}
$$

Since every operator acting in the Hilbert space of the atom (in particular the atomic density matrix $\rho_{\text {in }}^{a}$ ) is a linear combination of these four operators, this scheme allows us to determine the statistics of any atomic observable as a function of the measured probabilities for three values of the field-phase shift. This is equivalent to say that the atomic state can be reconstructed from the measurement.

A simple and common choice for the field state is a coherent one $|\alpha\rangle$. The coefficients $c, s$, and $d$ can be calculated very accurately by means of available analytical expressions [15]. To simplify the result as far as possible, the interaction time $\tau$ can be chosen such that $\lambda \tau \ll 1$, while the amplitude of the coherent state is high enough as to give a finite value for $\lambda \tau|\alpha|[16]$. In this case we are at the initial stages of the resonant evolution, long before the first collapse, and we have

$$
\begin{gathered}
c \simeq \cos ^{2}(\lambda \tau|\alpha|), \\
s \simeq \sin ^{2}(\lambda \tau|\alpha|), \\
d \simeq e^{i \theta} \sin (2 \lambda \tau|\alpha|),
\end{gathered}
$$

where $\theta$ is the phase of the coherent complex amplitude $\alpha$. Such a limit corresponds to the semiclassical atom-field interaction. The previous conditions are tantamount to considering that the atom-field interaction is governed by the Hamiltonian

$$
H=\omega S_{z}+\lambda\left(\alpha^{*} e^{i \omega t} S_{-}+\alpha e^{-i \omega t} S_{+}\right),
$$

where $\alpha$ is a classical complex amplitude.

The atomic state determination this scheme provides can be regarded as equivalent to the tomographic procedure used for the reconstruction of field states [2]. State reconstruction for finite-dimensional systems has been studied before [17]. It has been shown that the elements of the density matrix of an arbitrary spin $j$ are completely determined by the measurement of the spin projection along $4 j+1$ different directions. The procedure followed in this section corresponds to $j=\frac{1}{2}$. In the semiclassical limit, the measurement of the atomic population $S_{z}$ after the field phase shifts and the resonant interaction is in fact the measurement on the initial state of the three spin projections $S_{r}$,

$$
\begin{aligned}
S_{r}= & \cos (2 \lambda \tau|\alpha|) S_{z}+\sin (2 \lambda \tau|\alpha|) \sin \left(\delta_{r}+\theta\right) S_{x} \\
& +\sin (2 \lambda \tau|\alpha|) \cos \left(\delta_{r}+\theta\right) S_{y},
\end{aligned}
$$

where $S_{x}=(|e\rangle\langle g|+| g\rangle\langle e|) / 2$ and $S_{y}=i(|g\rangle\langle e|-| e\rangle\langle g|) / 2$ are the spin operators associated with the two-level atom.

Finally, when $\rho_{\text {in }}^{a}$ instead of $\rho_{\text {in }}^{f}$ is known in advance, the schemes studied in this work become measurements of field observables. This possibility has been already studied for the arrangements considered in Secs. II [18] and III [4].

\section{CONCLUSIONS}

We have found the atom-field observables that can be determined from some available measurements after resonant interaction. The dependence of the arrangement on controllable parameters, like phase shifts, can be used to deduce the statistics of different observables.

In particular, the two arrangements considered in this work provide the statistics of the relative phase between the atomic dipole and the field, as it could be expected from classical arguments. Moreover, atomic deflection provides a direct measurement of the relative-phase operator.

When the field state is known, we have a generalized measurement of atomic observables. It turns out that it provides the statistics of any observable allowing the reconstruction of the internal state of the atom.
[1] C. W. Helstrom, Quantum Detection and Estimation Theory (Academic, New York, 1976); A. Peres, Quantum Theory: Concepts and Methods (Kluwer, Dordrecht, 1993); A. Peres, Found. Phys. 20, 1441 (1990); H. P. Yuen, Phys. Lett. A 91, 101 (1982).

[2] K. Vogel and H. Risken, Phys. Rev. A 40, 2847 (1989); D. T. Smithey, M. Beck, M. G. Raymer, and A. Faridani, Phys. Rev. Lett. 70, 1244 (1993).

[3] M. J. Holland, D. F. Walls, and P. Zoller, Phys. Rev. Lett. 67, 1716 (1991); A. M. Herkommer, V. M. Akulin, and W. P. Schleich, ibid. 69, 3298 (1992); P. Domokos, P. Adam, J. Janszky, and A. Zeilinger, ibid. 77, 1663 (1996).

[4] M. Freyberger and A. M. Herkommer, Phys. Rev. Lett. 72,
1952 (1994); B. Baseia, R. Vyas, C. M. A. Dantas, and V. S. Bagnato, Phys. Lett. A 194, 153 (1994).

[5] A. Luis and L. L. Sánchez-Soto, Opt. Commun. 133, 159 (1997).

[6] A. Luis and L. L. Sánchez-Soto, Phys. Rev. A 56, 994 (1997).

[7] R. Lynch, Phys. Rep. 256, 367 (1995).

[8] B. W. Shore and P. L. Knight, J. Mod. Opt. 40, 1195 (1993).

[9] M. Brune, S. Haroche, V. Lefevre, J. M. Raimond, and N. Zagury, Phys. Rev. Lett. 65, 976 (1990); H. Paul, Quantum Opt. 3, 169 (1991).

[10] K. E. Cahill and R. J. Glauber, Phys. Rev. 177, 1882 (1969); F. T. Arecchi, E. Courtens, R. Gilmore, and H. Thomas, Phys. Rev. A 6, 2211 (1972); A. Perelomov, Generalized Coherent 
States and their Applications (Springer-Verlag, Berlin, 1986).

[11] P. Meystre, E. Schumacher, and S. Stenholm, Opt. Commun. 73, 443 (1989).

[12] M. Grabowski, Int. J. Theor. Phys. 28, 1215 (1989).

[13] U. Leonhardt and H. Paul, Phys. Rev. A 48, 4598 (1993); T. Kiss, U. Herzog, and U. Leonhardt, ibid. 52, 2433 (1995).

[14] G. Rempe, H. Walther, and N. Klein, Phys. Rev. Lett. 58, 353 (1987); G. Rempe, F. Schmidt-Kaler, and H. Walther, ibid. 64, 2783 (1990); M. Brune, P. Nussenzveig, F. Schmidt-Kaler, F. Bernardot, A. Maali, J. M. Raimond, and S. Haroche, ibid. 72, 3339 (1994); L. Davidovich, M. Brune, J. M. Raimond, and S. Haroche, Phys. Rev. A 53, 1295 (1996).

[15] J. I. Cirac and L. L. Sánchez-Soto, Phys. Rev. A 40, 3743
(1989); M. Fleischhauer and W. Schleich, ibid. 47, 4258 (1993).

[16] S. M. Dutra, P. L. Knight, and H. Moya-Cessa, Phys. Rev. A 48, 3168 (1993); S. M. Dutra and P. L. Knight, ibid. 49, 1506 (1994).

[17] R. G. Newton and B. Young, Ann. Phys. (N.Y.) 49, 393 (1968); W. Band and J. L. Park, Found. Phys. 1, 133 (1970); J. L. Park and W. Band, ibid. 1, 211 (1971); W. Band and J. L. Park, ibid. 1, 339 (1971); H. M. Wiseman, Quantum Semiclassic. Opt. 7, 569 (1995).

[18] P. J. Bardroff, E. Mayr, and W. P. Schleich, Phys. Rev. A 51, 4963 (1995). 